\section{(6) OPEN ACCESS}

\title{
Association of circulating miR-223 and miR-16 with disease activity in patients with early rheumatoid arthritis
}

\author{
Mária Filková, ${ }^{1}$ Borbala Aradi, ${ }^{1}$ Ladislav Šenolt, ${ }^{2}$ Caroline Ospelt, ${ }^{1}$ Serena Vettori, ${ }^{1}$ \\ Heřman Mann, ${ }^{2}$ Andrew Filer, ${ }^{3}$ Karim Raza, ${ }^{3}$ Christopher D Buckley, ${ }^{3}$ Martyn Snow, ${ }^{4}$ \\ Jiř́ Vencovský, ${ }^{2}$ Karel Pavelka, ${ }^{2}$ Beat A Michel, ${ }^{1}$ Renate E Gay, ${ }^{1}$ Steffen Gay, ${ }^{1}$ \\ Astrid Jüngel $^{1}$
}

\begin{abstract}
Handling editor Tore K Kvien
- Additional material is published online only. To view please visit the journal online (http://dx.doi.org/10.1136/ annrheumdis-2012-202815).

${ }^{1}$ Centre of Experimental Rheumatology, University Hospital Zurich, Zurich, Switzerland

${ }^{2}$ Department of Clinical and Experimental Rheumatology of the 1st Faculty of Medicine, Institute of Rheumatology, Charles University in Prague, Prague, Czech Republic ${ }^{3}$ University of Birmingham, Birmingham, UK

${ }^{4}$ Royal Orthopaedic Hospital Birmingham NHS Foundation Trust, Birmingham, UK
\end{abstract}

\section{Correspondence to} Dr A Jüngel, Centre of Experimental Rheumatology, University Hospital Zurich, Gloriastrasse 23, Zurich CH-8091, Switzerland; astrid.juengel@usz.ch

Received 11 October 2012 Revised 8 July 2013 Accepted 9 July 2013 Published Online First 29 July 2013

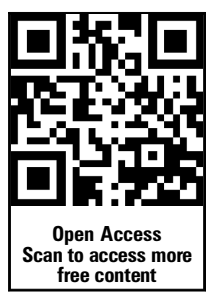

CrossMark

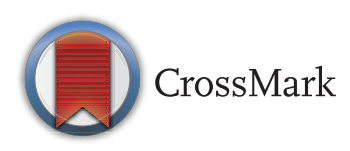

To cite: Filková $M$, Aradi $B$, Šenolt $\mathrm{L}$, et al. Ann Rheum Dis 2014;73:1898-1904.

\section{ABSTRACT}

Background Identification of parameters for early diagnosis and treatment response would be beneficial for patients with early rheumatoid arthritis (ERA) to prevent ongoing joint damage. miRNAs have features of potential biomarkers, and an altered expression of miRNAs was shown in established rheumatoid arthritis (RA).

Objective To analyse RA associated miRNAs in the sera of patients with ERA to find markers of early disease, clinical activity or predictors of disease outcome.

Methods Total RNA was isolated from whole sera in ERA patients (prior to and after 3 and 12 months of therapy with disease modifying antirheumatic drugs), in patients with established RA and in healthy controls ( $\mathrm{HC}$ ) using phenol-chloroform extraction. Expression of miR-146a, miR-155, miR-223, miR-16, miR-203, miR-132 and miR-124a was analysed by TaqMan Real Time PCR. Results From all analysed miRNAs, levels of miR-146a, miR-155 and miR-16 were decreased in the sera of ERA patients in comparison with established RA. A change in circulating miR-16 in the first 3 months of therapy was associated with a decrease in DAS28 in long term followup in ERA ( $p=0.002$ ). Levels of circulating miR-223 in treatment naïve ERA correlated with $C$ reactive protein $(p=0.008)$, DAS28 $(p=0.031)$ and change in DAS28 after 3 months $(p=0.003)$ and 12 months $(p=0.011)$ of followup. However, neither miR-16 nor miR-223 could distinguish ERA from $\mathrm{HC}$.

Conclusions Differential expression of circulating miR146a, miR-155 and miR-16 in the sera of ERA patients may characterise an early stage of the disease. We suggest miR-223 as a marker of disease activity and miR-16 and miR-223 as possible predictors for disease outcome in ERA.

\section{INTRODUCTION}

Rheumatoid arthritis (RA) is a systemic, inflammatory, autoimmune disorder with progressive articular damage that may result in severe lifelong disability. A considerable number of RA patients develop severe disability early in the disease. ${ }^{1}$ Studies have shown a beneficial effect of early treatment on clinical outcome and reduction in joint damage that prevents irreversible joint destruction and disability. ${ }^{2-4}$ In addition, a delay of a few months from the onset of symptoms to the institution of therapy decreases the ability of the traditional single drug strategy to induce remission in early rheumatoid arthritis (ERA). ${ }^{5-7}$ Therefore, in order to define patients at earlier stages of the disease who would benefit from early effective intervention, new RA classification criteria were developed. ${ }^{8}$ However, while some patients profit from initial monotherapy, others fail to demonstrate clinical or radiological responses, even to combination therapy. ${ }^{9}$ Until now, several predictors of response to disease modifying antirheumatic drugs (DMARD) and biological treatments such as cytokine profiles or gene expression analysis have been described but are either impractical or lack specificity to be adopted in daily clinical practice. $^{9}{ }^{10}$ Therefore, it is important to find parameters that identify patients with early arthritis and to find predictors of treatment response in ERA to optimise individual management of early arthritis based on the expected disease course and to prevent over or under treatment. ${ }^{8-10}$

miRNAs are short single stranded non-coding RNAs involved in the post-transcriptional regulation of gene expression. ${ }^{11}$ Altered expression of miRNAs has been described under various pathological conditions, including rheumatic and other autoimmune diseases. $^{12} 13$ We and others reported that miR-146a, miR-155 and miR-203 are overexpressed while miR-124a appeared suppressed in RA synovial fibroblasts. ${ }^{14-17}$ Dysregulation of miR-146a, miR-155, miR-223, miR-16 and miR-132 was observed in immune cells derived from patients with RA. ${ }^{14}{ }^{18-22}$ As miRNAs are stably present in cell free form in body fluids and circulating miRNAs were shown to have signatures related to tumour classification and disease progression, they are becoming new candidate biomarkers for diagnosis and prognosis in different diseases. ${ }^{23-25}$ Although expression of several miRNAs has recently been analysed in plasma and synovial fluid of patients with established RA and osteoarthritis, ${ }^{26}$ there are no reports to date on the important phase of ERA.

The aim of our study was to analyse the profile of selected RA related cell free circulating miRNAs in the sera of patients with ERA prior to and after therapy with DMARD, in established RA and in healthy controls (HC). Using this approach, we hoped to identify potential tools distinguishing early and late phases of the disease, markers of 
clinical activity or predictors of disease outcome. In addition, we analysed intracellular expression of these miRNAs in synovial fibroblasts from patients with ERA.

\section{MATERIAL AND METHODS}

\section{Collection of sera and characteristics of patients with RA}

Sera and clinical details from 34 patients with ERA who fulfilled the 2010 American College of Rheumatology (ACR)/European League Against Rheumatism (EULAR) classification criteria for $\mathrm{RA}^{8}$ with a symptom duration $<8$ months were obtained at baseline, and at 3 and 12 months after treatment. Patients with ERA were recruited from the outpatient clinic of the Institute of Rheumatology, Prague. Sera from 28 patients with established treated RA of disease duration 9.28 $\mathbf{6 . 5 2}$ years who fulfilled the 1987 revised ACR criteria for the classification of $\mathrm{RA}^{27}$ were obtained from both the Department of Rheumatology, University Hospital, Zurich, and the Institute of Rheumatology, Prague. Clinical characteristics of the patients and the $16 \mathrm{HC}$ (University Hospital, Zurich) are shown in table 1. Written informed consent was obtained from all participants, and the study was approved by the local ethics committees.

\section{Synovial fibroblasts from patients with inflammatory arthritis}

Synovial fibroblasts (SF) were isolated from synovial tissues obtained during ultrasound guided synovial tissue biopsy (University of Birmingham, UK). Synovial tissue samples were taken from patients with untreated ERA $(n=7)$ with a disease duration < 13 months who met the 1987 ACR criteria, ${ }^{27}$ from patients with knee pain of non-inflammatory origin $(n=9)$ and from patients with resolving arthritis different from RA $(n=14)$. Written informed consent was obtained from each donor, and the study was approved by the local ethics committee. For a detailed description of the methods, see online supplementary material.

Expression of miRNAs was analysed by TaqMan Real Time PCR. The $\mathrm{dCt}$ method was used for relative quantification of miRNA. dCt was calculated as follows: Ct (let-7a) - Ct (miRNA of interest) and therefore higher $\mathrm{dCt}$ values represent higher expression. The data summarising the establishment of the method for the detection of miRNAs in sera are given in the online supplementary material (see online supplementary figure 1).

\section{RESULTS}

\section{Circulating miRNAs are differentially expressed in the sera of patients with ERA and established RA}

As several miRNAs have been reported to show altered expression in immune cells, SF, plasma or synovial fluid from patients with established RA, we anticipated dysregulation of circulating miRNAs at the onset of the disease in comparison with the established state. Considering circulating miRNAs as potential biomarkers, their analysis in sera would be of interest due to easy accessibility of samples for clinical use. Therefore, analysis of RA related miR-146a, miR-155, miR-203, miR-124a, miR-223, miR-16 and miR-132 in sera was performed in patients with treatment naïve ERA, established treated RA and HC.

Levels of miR-146a were significantly lower in the sera of patients with ERA (dCt $0.44 \pm 0.93$ ) in comparison with patients with established RA (dCt 1.20 $\pm 1.00, \mathrm{p}<0.01)$ or $\mathrm{HC}(\mathrm{dCt}$ $1.27 \pm 1.07, \mathrm{p}<0.05)$. There was no difference between established RA and HC (figure 1A). Circulating miR-155 in ERA patients $(\mathrm{dCt}-6.31 \pm 1.05)$ showed comparable levels with $\mathrm{HC}$ (dCt $-5.84 \pm 1.24)$ while it significantly differed from patients with established RA who demonstrated higher levels of miR-155 $(\mathrm{dCt}-4.68 \pm 1.86, \mathrm{p}<0.001)$ (figure 1B). Similarly, miR-16 was

Table 1 Characteristics of patients with early and established rheumatoid arthritis, and healthy controls, whose sera were used for analysis of expression of circulating miRNAs in the present study

\begin{tabular}{|c|c|c|c|c|c|}
\hline & Early RA & & & Established RA & Healthy controls \\
\hline Sex (F/M) & $25 / 9$ & & & $17 / 9$ & $9 / 7$ \\
\hline Age (years) & $47.30 \pm 14.41$ & & & $57.89 \pm 13.29$ & $39.00 \pm 14.12$ \\
\hline Disease duration & $<8$ months & & & $9.28 \pm 6.52$ years & 0 \\
\hline Treatment & Baseline & 3 months & 12 months & & \\
\hline Methotrexate & 0 & 28 & 28 & 14 & 0 \\
\hline Sulfasalazine & 0 & 4 & 4 & 2 & 0 \\
\hline Leflunomide & 0 & 1 & 1 & 8 & 0 \\
\hline Glucocorticoids & 0 & 30 & 30 & 6 & 0 \\
\hline Biologics & 0 & 0 & 0 & 19 & 0 \\
\hline Clinical characteristics & Baseline & 3 months & 12 months & & \\
\hline $\mathrm{n}$ & 31 & 31 & 32 & 28 & 16 \\
\hline RF positivity (\%) & 64.52 & NA & 66.67 & 85.71 & NA \\
\hline $\lg \mathrm{RF}(\mathrm{U} / \mathrm{mL})$ & $31.79 \pm 42.86$ & NA & $19.83 \pm 17.48$ & NA & NA \\
\hline $\operatorname{lgM} \mathrm{RF}(\mathrm{U} / \mathrm{mL})$ & $78.01 \pm 225.50$ & NA & $84.89 \pm 186.30$ & NA & NA \\
\hline $\lg A R F(U / m L)$ & $59.14 \pm 102.70$ & NA & $36.54 \pm 79.37$ & NA & NA \\
\hline ACPA positivity (\%) & 51.61 & NA & 48.48 & 85.71 & NA \\
\hline ACPA (mmol/L) & $1018 \pm 1871$ & NA & $973 \pm 1600$ & NA & \\
\hline DAS28 & $5.41 \pm 1.61$ & $2.60 \pm 1.21^{* \star *}$ & $2.54 \pm 1.18^{* \star \star}$ & $4.10 \pm 1.32$ & NA \\
\hline CRP (mg/L) & $18.36 \pm 23.30$ & $4.03 \pm 6.60^{* * *}$ & $2.77 \pm 2.57^{* * *}$ & $21.05 \pm 37.58$ & NA \\
\hline $\operatorname{ESR}(\mathrm{mm} / \mathrm{h})$ & $31.00 \pm 19.52$ & $15.09 \pm 9.87^{* * *}$ & $11.43 \pm 7.71^{* * *}$ & NA & NA \\
\hline Leucocytes $\times 10^{9} / \mathrm{mm}^{3}$ & $8.34 \pm 2.53$ & $7.41 \pm 1.74$ & $6.70 \pm 2.72^{* *}$ & NA & NA \\
\hline
\end{tabular}

Data are expressed as the mean \pm SD.

${ }^{*} p<0.05,{ }^{* *} p<0.01,{ }^{* *} p<0.001$ compared with baseline.

ACPA, anticitrullinated proteins antibodies; CRP, C reactive protein; DAS28, disease activity score calculated with ESR; ESR, erythrocyte sedimentation rate; NA, not analysed; RA

rheumatoid arthritis; RF, rheumatoid factor. 

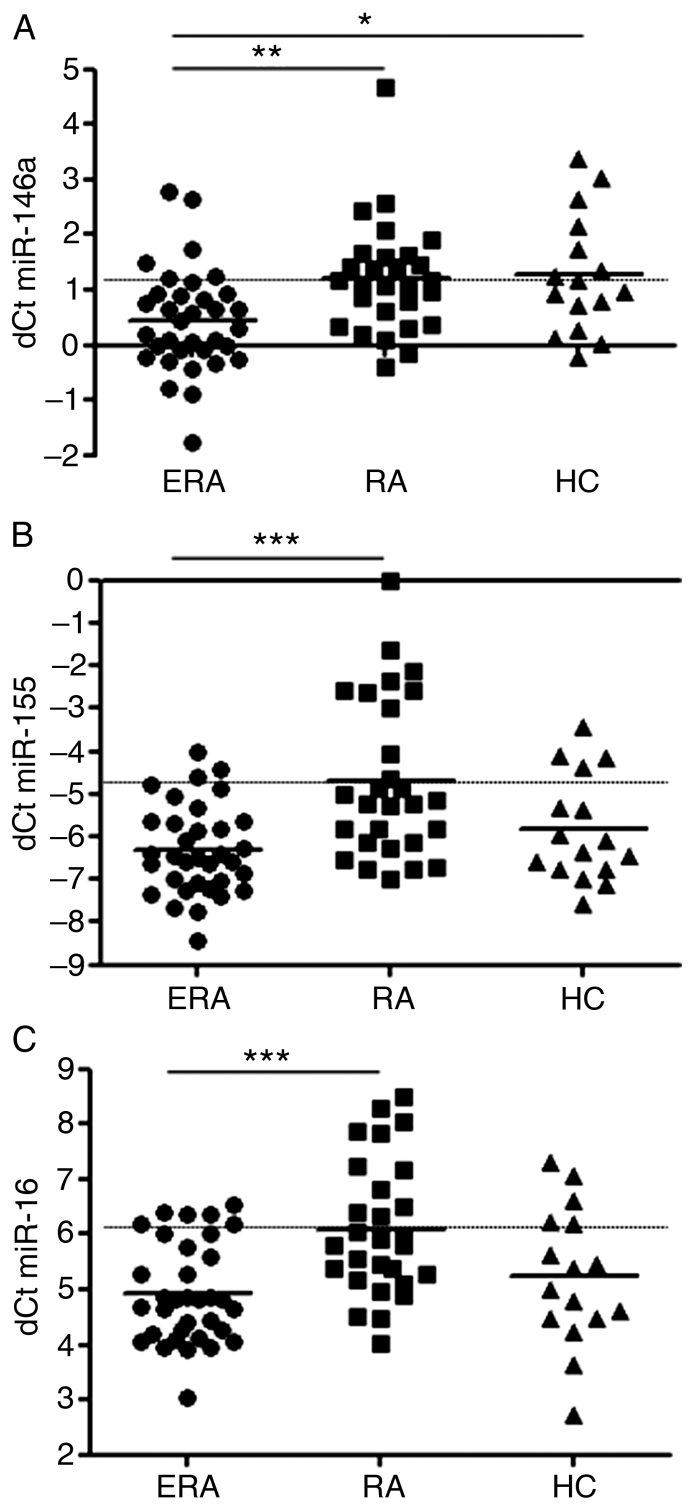

Figure 1 Comparison of levels of circulating miR-146a (A), miR-155 (B) and miR-16 (C) in the sera of patients with treatment naive early rheumatoid arthritis (ERA), established rheumatoid arthritis (RA) and healthy controls $(\mathrm{HC}) .{ }^{*} \mathrm{p}<0.05,{ }^{* *} \mathrm{p}<0.01,{ }^{* * *} \mathrm{p}<0.001$. dCt was calculated as follows: Ct (let-7a) - Ct (miRNA of interest) and therefore higher $\mathrm{dCt}$ values represent higher levels. Broken line refers to levels of miRNAs in established RA.

found at significantly lower levels in the sera of patients with ERA (dCt $4.93 \pm 0.91)$ than in patients with established RA ( $\mathrm{dCt}$ $6.10 \pm 1.22, \mathrm{p}<0.001)$ while it was comparable with $\mathrm{HC}(\mathrm{dCt}$ $5.23 \pm 1.25$ ). The levels of miR-16 in established RA were higher than in HC but did not reach statistical significance (figure 1C).

The patterns of miR-132 and miR-223 were not different in ERA, established RA and HC (dCt $-4.31 \pm 0.90,-3.76 \pm 1.07$, $-4.41 \pm 1.23$ for miR-132; dCt $5.67 \pm 1.04,6.23 \pm 1.45,5.55$ \pm 0.77 for miR-223). MiR-124a and miR-203 in sera were present in negligible amounts close to the detection limit and were therefore excluded from further analysis (data not shown).

\section{Change in circulating miR-16 during therapy is associated with decreasing disease activity}

Subsequently, the effect of therapy with DMARDs on levels of miRNAs was explored in ERA patients. Also, the association of circulating miRNAs with markers of disease activity in ERA and established RA was evaluated. Follow-up within 3 months (M3) after initiation of therapy appeared to be a very important time point with respect to clinical response and prediction of outcome at 12 months (M12). ${ }^{28} 29$

Levels of miR-16 increased by $34.31 \%$ in $24 / 34$ ERA patients after 3 months of therapy. Although miR-16 was the only miRNA analysed in our study, showing a trend towards higher levels after 3 months of treatment with DMARDs (dCt $4.93 \pm 0.91$ vs $5.71 \pm 1.27$ ), it did not reach statistical significance in the whole group of ERA patients. However, levels of miR-16 at M12 (dCt 3.85 \pm 1.37 ) significantly decreased in comparison with both baseline $(\mathrm{p}<0.05)$ and M3 $(\mathrm{p}<0.001$, figure $2 \mathrm{~A})$. In addition, the increase in miR-16 at M3 was verified by an independent method using TaqMan Low Density Arrays cards (TLDAs) (3.768-fold change, see online supplementary table 1). The effect of different treatment modalities on levels of miR-16 is given in the online supplementary table 2 .

Higher levels of miR-16 at baseline correlated with a greater improvement in disease activity, represented by a larger decrease in DAS28 from baseline to M3 $(r=-0.478, p=0.008)$ (figure 2B). Similarly, levels of miR-16 at M3 correlated with a change in disease activity $\triangle \mathrm{DAS} 28$ from M3 to M12 $(\mathrm{r}=-0.355$, $\mathrm{p}=0.047$ ) (figure 2C). Most importantly, the change in levels of miR-16 from baseline to $\mathrm{M} 3$ were negatively associated with the change in DAS28 from M3 to M12 $(r=-0.519, p=0.002)$ (figure 2D) suggesting that the increase in circulating miR-16 within the first 3 months after treatment initiation was followed by the decrease in disease activity in the subsequent 9 months. Interestingly, levels of miR-16 at baseline negatively correlated with levels of anticitrullinated protein antibodies (ACPA) $(r=$ $-0.365, p=0.037)$. However, levels of miR-16 did not correlate with $\mathrm{C}$ reactive protein (CRP), erythrocyte sedimentation rate or IgG, IgM or IgA rheumatoid factors (RF) at baseline, M3 or M12 (data not shown).

Although differentially expressed in ERA sera in comparison with established RA sera, levels of circulating miR-16 showed no association with disease activity in established RA (data not shown).

MiR-223 as a marker of disease activity in treatment naïve ERA and its association with disease outcome during short and long time follow-up

Levels of miR-223 were found to be decreased by $17.65 \%$ in 24/34 ERA patients 3 months after treatment initiation. However, in the group of all ERA patients, the change in levels of miR-223 at M3 in comparison with baseline did not reach statistical significance (dCt $5.67 \pm 0.104$ vs $5.12 \pm 0.91$ ). In fact, after 12 months of treatment, levels of miR-223 (dCt $4.34 \pm 0.78)$ significantly decreased in comparison with levels at baseline $(\mathrm{p}<0.001)$ and at M3 $(\mathrm{p}<0.01)$ (figure 3A). Further analysis using TLDA cards showed no change in miR-223 at M3 (1.065-fold change) but confirmed the decreased levels of miR-223 at M12 (0.692-fold change, see online supplementary table 1). The effect of different treatment modalities on levels of miR-223 is given in the online supplementary table 2 .

In ERA prior to treatment initiation, levels of miR-223 positively correlated with CRP $(r=0.519, p=0.008$ figure $3 \mathrm{~B})$ and with DAS28 ( $\mathrm{r}=0.389, \mathrm{p}=0.031) \quad$ (figure $3 \mathrm{C})$ at baseline. Importantly, levels of miR-223 at baseline correlated with the decrease in DAS28 from baseline to M3 $(r=-0.522, p=0.003)$ (figure 3D) as well as from baseline to M12 ( $\mathrm{r}=-0.460$, $\mathrm{p}=0.011$ ) (figure 3E). Moreover, the greater decrease in levels of miR-223 from baseline to M12, the more improvement in 
A
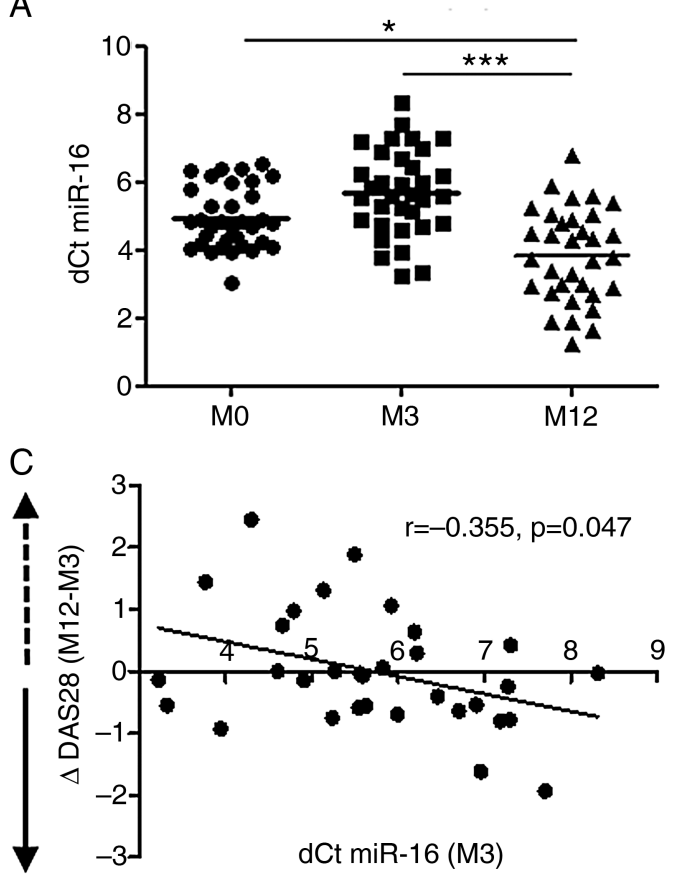

B
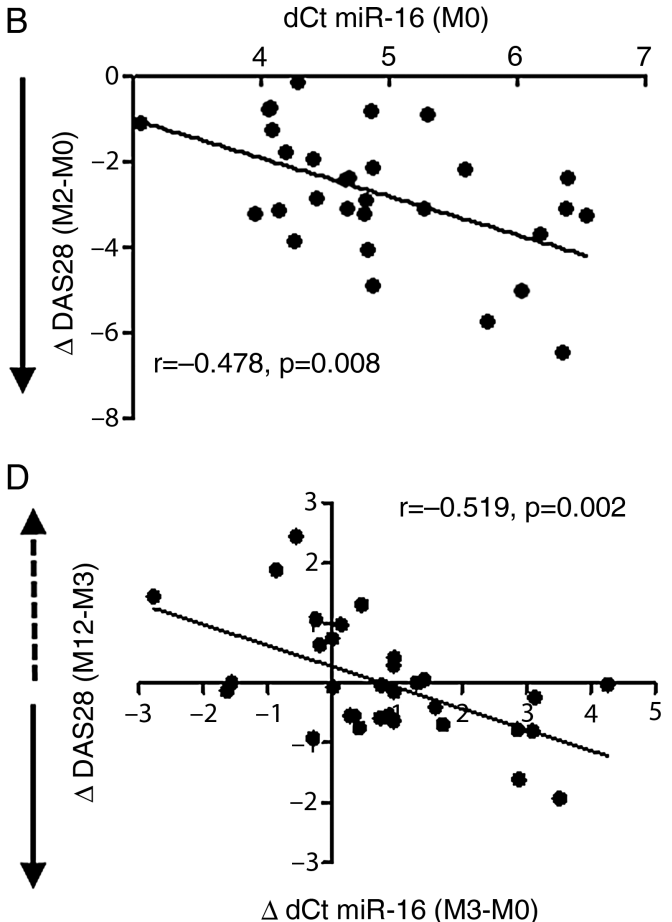

Figure 2 Change in levels of circulating miR-16 in the sera of patients with early rheumatoid arthritis (ERA) from baseline (M0) to 3 (M3) and 12 (M12) months after treatment with disease modifying antirheumatic drugs (A). ${ }^{*} \mathrm{p}<0.05$, ${ }^{* * *} \mathrm{p}<0.001$. Levels of miR-16 (dCt) at baseline (M0) correlated with change in DAS28 ( $\triangle D A S 28$ ) from M0 to M3 (B). Levels of miR-16 in ERA sera at M3 correlated with $\triangle D A S 28$ from M3 to M12 (C). Importantly, the change in levels of miR-16 ( $\Delta \mathrm{dCt}$ ) from M0 to M3 was negatively associated with $\triangle \mathrm{DAS} 28$ from M3 to M12 (D). $\mathrm{dCt}$ was calculated as follows: $\mathrm{Ct}$ (let-7a) $-\mathrm{Ct}$ (miR-16) and therefore higher dCt values represent higher levels. The increase/decrease in $\triangle \mathrm{DAS} 28$ is indicated by a broken/full arrow, respectively.

DAS28 was observed after 12 months of therapy $(r=0.483$, $p=0.006$ ) (figure $3 F$ ). In contrast with significant correlations of miR-223 with disease activity in ERA patients before treatment, levels of miR-223 did not correlate with parameters of disease activity (DAS28 or CRP), or levels of ACPA, IgG, IgM or IgA RF at M3 or M12 after initiation of therapy (data not shown). In established RA, levels of miR-223 showed no correlation with disease activity (data not shown).

In addition, neither miR-146a nor miR-155 or miR-132 showed any association with disease activity in patients with ERA at baseline, M3 or M12, or in patients with established RA or any change in expression after therapy in ERA (data not shown).

\section{Decrease in levels of miR-223 in sera follows change in peripheral leucocyte count}

We found a significant correlation between baseline levels of miR-223 and peripheral leucocyte (PL) count in patients with ERA ( $r=0.459, p=0.007)$ (figure 4A). Therefore, we were interested in whether the change in levels of miR-223 after treatment followed the change in PL count. In fact, the larger decrease in miR-223 between baseline and M3 positively correlated with the decrease in PL count between these two time points $(r=0.397, p=0.025)$ (figure 4B). Also, the changes in levels of miR-223 and PL count within the whole period of 12 months correlated significantly $(\mathrm{r}=0.621, \mathrm{p}=0.0001)$ (figure $4 \mathrm{C})$. This suggests that the decrease in miR-223 in the sera of ERA patients after therapy is very likely attributable to the change in the number of PLs.

\section{Expression of miRNAs in synovial fibroblasts from patients with ERA}

Based on different levels of miRNAs in ERA reported here, we anticipated dysregulation of miRNAs in SF from patients with ERA. Therefore, expression of miR-146a, miR-155, miR-203, miR-124a, miR-223, miR-16 and miR-132 was analysed in isolated SF cultured in vitro.

In contrast with differences in levels of miRNAs observed in sera, there were no significant differences in expression of miRNAs analysed in our study among SF from patients with ERA, resolving arthritis of different disease entities as an example of non-RA arthritis and controls with noninflammatory arthralgia (see online supplementary figure 2)

\section{DISCUSSION}

RA is a chronic inflammatory disease characterised by progressive joint destruction. We and others have shown that altered expression of miRNAs in immune and resident cells involved in the pathogenesis of RA contributes to maintenance of pathognomic features typical of RA. ${ }^{13}$ For example, miR-155 and miR-146a are induced in response to inflammatory stimuli and are overexpressed in RA SF in comparison with osteoarthritis SF. ${ }^{14} 1830$ MiR-155 acts as a positive regulator and miR-146a as a negative regulator of inflammation in RA, as shown in vivo. ${ }^{18} 3132$ MiR-223 was shown to be overexpressed in synovial tissue of RA patients and, importantly, silencing of miR-223 reduced disease severity in experimental arthritis. ${ }^{33}$

The evidence of high stability of miRNAs in body fluids due to incorporation into microvesicles or stabilisation in complexes with RNA binding proteins (Argonaute 2, high density lipoproteins, nucleophosmin 1 ) and their easy accessibility make miRNA 

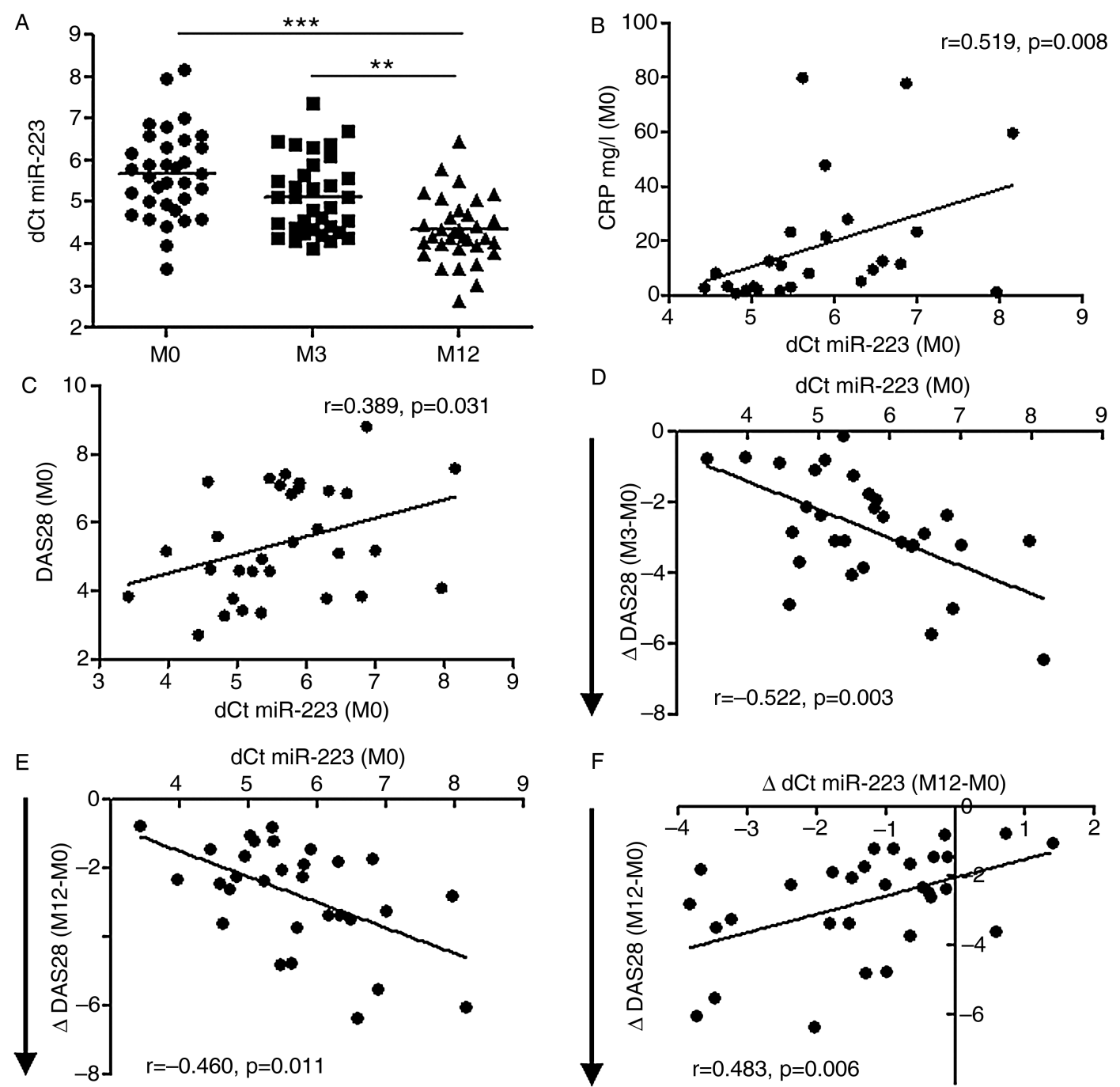

Figure 3 Change in levels of circulating miR-223 in the sera of patients with early rheumatoid arthritis (ERA) from baseline (M0) to 3 (M3) and 12 (M12) months after treatment with disease modifying antirheumatic drugs (A). ${ }^{*} p<0.01,{ }^{* * *} p<0.001$. Levels of circulating miR-223 (dCt) at baseline (M0) in the sera of ERA patients correlated with the levels of $C$ reactive protein (CRP) (B) and disease activity (DAS28) (C) at M0. Levels of circulating miR-223 at M0 correlated with the change in DAS28 ( $D A S 28)$ from M0 to M3 (D) as well as M12 (E). Change in levels of miR-223 from M0 to M12 correlated with $\triangle \mathrm{DAS} 28$ from M0 to M12 (F). dCt was calculated as follows: Ct (let-7a) - Ct (miR-223) and therefore higher dCt values represent higher levels. The decrease in $\triangle \mathrm{DAS} 28$ is indicated by a full arrow.

ideal biomarkers. ${ }^{23} 24{ }^{34-36}$ Moreover, changes in the number of miRNAs originating from blood cells or tissues and their specific profiles influenced by pathophysiological conditions, such as sepsis, have been described previously. ${ }^{37}$ Based on these data, we anticipated that levels of circulating miRNAs in sera would be different in patients with ERA and established RA.

We report here for the first time reduced levels of miR-146a, miR-155 and miR-16 in the sera of patients with ERA in comparison with patients with established RA. Levels of these miRNAs were comparable between patients with established RA and HC, similar to the data published by Murata et al, ${ }^{26}$ except for miR-132 that was shown to be lower in RA than in HC. ${ }^{26}$ Although intracellular expression of miR-146a and miR-155 was shown to be induced by the proinflammatory milieu in vitro, ${ }^{14} 18$ it is possible that cell free circulating miRNAs in sera do not necessarily reflect levels in the intracellular compartment. In fact, release of miRNAs may be selective due to the existence of a cellular selection mechanism for miRNA release and therefore the extracellular and cellular miRNA profiles may differ. ${ }^{38}$ Secretory cell free miRNAs were shown to transfer inhibitory signals to recipient cells. ${ }^{39}$ Although the functional role and impact of circulating miRNAs in living organisms remains currently unknown, we speculate that in ERA, miR-146a and miR-155 may be extensively taken up by recipient cells where they exert their regulatory activity. We propose that low levels of miR-146a, miR-155 and miR-16 in ERA may represent the early phase of RA. We hypothesise that progression of disease severity, disease duration, effect of treatment or other yet unknown factors can modulate levels of circulating miRNAs in established RA.

MiR-16 was reported to be overexpressed in peripheral blood mononuclear cells of RA patients with active disease in comparison with RA patients with low disease activity or with $\mathrm{HC}^{20}$ We showed here that the higher levels of miR-16 in the sera of treatment naïve patients with ERA correlated with better improvement in disease activity during the 3 months of follow-up. This is important because most clinical responses in ERA are seen in the first 3 months of therapy, as shown in table 1 . In addition, change in levels of circulating miR-16 within the first 3 months after initiation of therapy is associated with disease outcome in the following 9 months. 
A

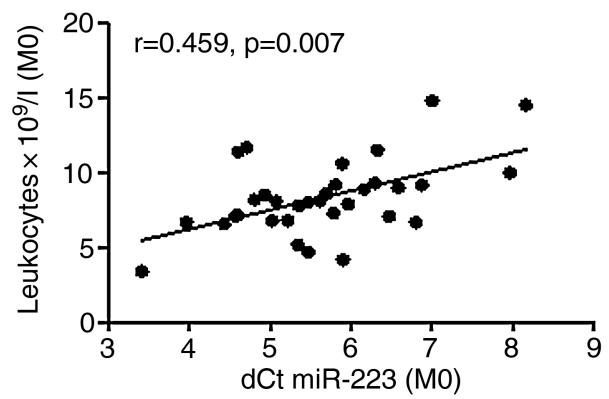

B

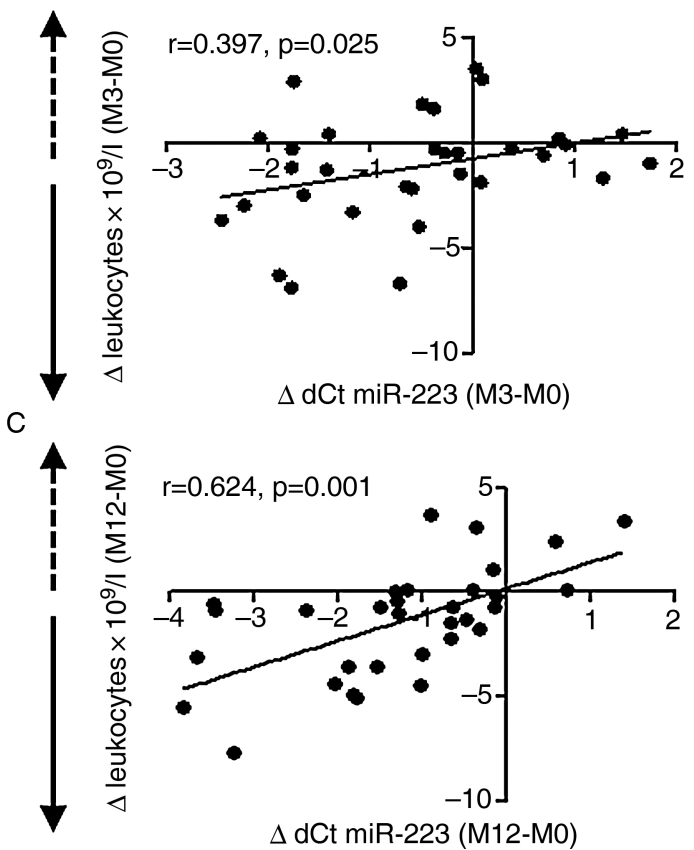

Figure 4 Levels of miR-223 at baseline (M0) correlated with peripheral leucocyte (PL) count at MO (A). Change in levels of circulating miR-223 ( $\Delta \mathrm{dCt}$ miR-223) from M0 to 3 months of follow-up (M3) correlated with changes in PL count between these two time points ( $\Delta$ leukocytes) in early rheumatoid arthritis patients (B). Similarly, $\Delta \mathrm{dCt}$ miR-223 from M0 to 12 months of follow-up (M12) correlated with $\Delta$ leukocytes from M0 to M12 (C). dCt was calculated as follows: $\mathrm{Ct}$ (let-7a) - Ct (miR-223) and therefore higher $\mathrm{dCt}$ values represent higher levels. The increase/decrease in $\Delta$ leukocytes is indicated by a broken/full arrow, respectively.

MiR-223 was recently demonstrated to be upregulated in peripheral $\mathrm{T}$ cells from patients with early as well as established RA compared with HC, but no correlation of miR-223 in these cells with DAS28, CRP, RF or ACPA was found in these patients. $^{22} 40$ In addition, no differences in expression of miR-223 in T cells were observed in untreated RA patients and those treated with glucocorticoids. ${ }^{22}$ Although not differentially expressed in the sera of ERA and RA patients, we suggest circulating cell free miR-223 as a marker of disease activity in treatment naïve patients with ERA based on significant associations with CRP and DAS28. At the same time, higher baseline levels of circulating miR-223 were associated with better improvement in disease activity in ERA after the therapeutic intervention. We suggest that levels of miR-16 and miR-223 may characterise patients with good/worse response to therapy.

In the study, we showed the changes in levels of miR-16 and miR-223 over time after initiation of therapy. We suggest that the decrease in miR-223 in ERA sera after therapy may be attributed to change in PL count. Accordingly, haematopoietic cells express miR-223 and are considered an important source of circulating miR-223. ${ }^{3741}$ Although the main source of circulating miRNAs is currently unknown, they can originate from heterogenous cells, such as circulating blood cells or cells in organs. Therefore, we speculate that differential levels of miR-16 and miR-223 in the sera of ERA patients after therapy may reflect altered expression in blood cells or solid tissues. ${ }^{13}$

Apart from the contribution of inflammatory cells abundantly present in the hyperplastic RA synovium, SF are believed to actively drive joint inflammation and destruction. ${ }^{42}{ }^{43}$ Although we observed different levels of miRNAs in ERA sera, we did not see any differences in SF from patients with ERA cultivated in vitro, possibly due to type II statistical error. As shown previously, ${ }^{26}$ fibroblast-like synoviocytes, mononuclear cells and synovial tissues secrete miRNAs in distinct patterns. A differential profile of cytokines influencing the microenvironment required for persistent RA has been observed in the synovial fluids of patients with early synovitis which developed later into RA. ${ }^{44}$ Also, distinct expression of miRNAs in synovial tissues in established RA, as a result of chronic exposure of SF to the inflammatory milieu accompanying RA, has been reported. ${ }^{14} 1833$ Therefore, our data obtained in vitro in isolated SF do not necessarily reflect the situation in synovial tissue. The association of miRNAs with specific immunohistochemical findings in synovial tissues at the early phase and how cell free miRNAs originating from a joint compartment contribute to the spectrum and levels of circulating miRNAs in blood serum/plasma remain to be investigated.

In conclusion, we have shown here for the first time low expression of miR-146, miR-155 and miR-16 in the sera of patients with early in comparison with established RA. Although further studies in larger patient cohorts are needed, our data support circulating miR-223 as a marker of disease activity in patients with treatment naïve ERA. Moreover, monitoring levels of miR-16 and miR-223 may become a useful tool to predict disease outcome in patients with ERA.

Acknowledgements The authors acknowledge Jitka Smékalová for technical assistance.

Funding This work was supported by IMI BTCure, IAR Epalinges, EU-TEAM, project No 023728 for conceptual development of research organisation by the Ministry of Health, Czech Republic, IGA project No NT 14498 and SVV project No 264511.

Competing interests None.

Ethics approval The study was approved by the ethics commission of Zurich, Kantonale Ethikkommission Zürich), the ethics committee at the Institute of Rheumatology, Charles University, Prague, Czech Republic and the ethics committee at University of Birmingham, UK.

Provenance and peer review Not commissioned; externally peer reviewed.

Open Access This is an Open Access article distributed in accordance with the Creative Commons Attribution Non Commercial (CC BY-NC 3.0) license, which permits others to distribute, remix, adapt, build upon this work non-commercially, and license their derivative works on different terms, provided the original work is properly cited and the use is non-commercial. See: http://creativecommons.org/ licenses/by-nc/3.0/

\section{REFERENCES}

1 Bukhari $M$, Harrison $B$, Lunt $M$, et al. Time to first occurrence of erosions in inflammatory polyarthritis: results from a prospective community-based study. Arthritis Rheum 2001;44:1248-53.

2 Bukhari MA, Wiles NJ, Lunt $M$, et al. Influence of disease-modifying therapy on radiographic outcome in inflammatory polyarthritis at five years: results from a large observational inception study. Arthritis Rheum 2003;48:46-53.

3 Raza K, Saber TP, Kvien TK, et al. Timing the therapeutic window of opportunity in early rheumatoid arthritis: proposal for definitions of disease duration in clinical trials. Ann Rheum Dis 2012;71:1921-3. 
4 Nell VP, Machold KP, Eberl G, et al. Benefit of very early referral and very early therapy with disease-modifying anti-rheumatic drugs in patients with early rheumatoid arthritis. Rheumatology (Oxford) 2004;43:906-14.

5 Möttönen T, Hannonen P, Korpela M, et al. Delay to institution of therapy and induction of remission using single-drug or combination-disease-modifying antirheumatic drug therapy in early rheumatoid arthritis. Arthritis Rheum 2002;46:894-8.

6 Emery P, Kvien TK, Combe B, et al. Combination etanercept and methotrexate provides better disease control in very early ( $<=4$ months) versus early rheumatoid arthritis ( $>4$ months and $<2$ years): post hoc analyses from the COMET study. Ann Rheum Dis 2012;71:989-92.

7 van der Linden MP, le Cessie S, Raza K, et al. Long-term impact of delay in assessment of patients with early arthritis. Arthritis Rheum 2010;62:3537-46.

8 Aletaha D, Neogi T, Silman AJ, et al. 2010 Rheumatoid arthritis classification criteria: an American College of Rheumatology/European League Against Rheumatism collaborative initiative. Arthritis Rheum 2010;62:2569-81.

9 Allaart CF, Huizinga TW. Treatment strategies in recent onset rheumatoid arthritis. Curr Opin Rheumatol 2011:23:241-4.

10 Mjaavatten MD, van der Heijde D, Uhlig T, et al. The likelihood of persistent arthritis increases with the level of anti-citrullinated peptide antibody and immunoglobulin M rheumatoid factor: a longitudinal study of 376 patients with very early undifferentiated arthritis. Arthritis Res Ther 2010;12:R76.

11 Filipowicz W, Bhattacharyya SN, Sonenberg N. Mechanisms of post-transcriptiona regulation by microRNAs: are the answers in sight? Nat Rev Genet 2008;9:102-14.

12 Furer $\mathrm{V}$, Greenberg JD, Attur $\mathrm{M}$, et al. The role of microRNA in rheumatoid arthritis and other autoimmune diseases. Clin Immunol 2010;136:1-15.

13 Filková M, Jüngel A, Gay RE, et al. MicroRNAs in rheumatoid arthritis: potential role in diagnosis and therapy. BioDrugs 2012;26:131-41.

14 Stanczyk J, Pedrioli DM, Brentano F, et al. Altered expression of MicroRNA in synovial fibroblasts and synovial tissue in rheumatoid arthritis. Arthritis Rheum 2008:58:1001-9.

15 Stanczyk J, Ospelt C, Karouzakis E, et al. Altered expression of microRNA-203 in rheumatoid arthritis synovial fibroblasts and its role in fibroblast activation. Arthritis Rheum 2011;63:373-81.

16 Nakamachi Y, Kawano S, Takenokuchi M, et al. MicroRNA-124a is a key regulator of proliferation and monocyte chemoattractant protein 1 secretion in fibroblast-like synoviocytes from patients with rheumatoid arthritis. Arthritis Rheum 2009;60:1294-304

17 Kawano S, Nakamachi Y. miR-124a as a key regulator of proliferation and MCP-1 secretion in synoviocytes from patients with rheumatoid arthritis. Ann Rheum Dis 2011;70(Suppl 1):i88-91.

18 Kurowska-Stolarska M, Alivernini S, Ballantine LE, et al. MicroRNA-155 as a proinflammatory regulator in clinical and experimental arthritis. Proc Natl Acad Sci USA 2011:108:11193-8.

19 Niimoto T, Nakasa T, Ishikawa M, et al. MicroRNA-146a expresses in interleukin-17 producing T cells in rheumatoid arthritis patients. BMC Musculoskelet Disord 2010;11:209.

20 Pauley KM, Satoh M, Chan AL, et al. Upregulated miR-146a expression in peripheral blood mononuclear cells from rheumatoid arthritis patients. Arthritis Res Ther 2008:10:R101.

21 Li J, Wan Y, Guo Q, et al. Altered microRNA expression profile with miR-146a upregulation in $\mathrm{CD} 4+\mathrm{T}$ cells from patients with rheumatoid arthritis. Arthritis Res Ther 2010:12:R81.

22 Fulci V, Scappucci G, Sebastiani GD, et al. miR-223 is overexpressed in T-lymphocytes of patients affected by rheumatoid arthritis. Hum Immunol 2010;71:206-11.
23 Weber JA, Baxter DH, Zhang $\mathrm{S}$, et al. The microRNA spectrum in 12 body fluids. Clin Chem 2010;56:1733-41.

24 Mitchell PS, Parkin RK, Kroh EM, et al. Circulating microRNAs as stable blood-based markers for cancer detection. Proc Natl Acad Sci USA 2008:105:10513-18.

25 Chen X, Ba Y, Ma L, et al. Characterization of microRNAs in serum: a novel class of biomarkers for diagnosis of cancer and other diseases. Cell Res 2008:18:997-1006.

26 Murata K, Yoshitomi H, Tanida S, et al. Plasma and synovial fluid microRNAs as potential biomarkers of rheumatoid arthritis and osteoarthritis. Arthritis Res Ther 2010;12:R86.

27 Arnett FC, Edworthy SM, Bloch DA, et al. The American Rheumatism Association 1987 revised criteria for the classification of rheumatoid arthritis. Arthritis Rheum 1988;31:315-24.

28 van der Heijde D, Keystone EC, Curtis JR, et al. Timing and magnitude of initial change in disease activity score 28 predicts the likelihood of achieving low disease activity at 1 year in rheumatoid arthritis patients treated with certolizumab pegol: a post-hoc analysis of the RAPID 1 trial. J Rheumatol 2012;39:1326-33.

29 Curtis JR, McVie T, Mikuls TR, et al. Clinical response within 12 weeks as a predictor of future low disease activity in patients with early RA: Results from the TEAR Trial. J Rheumatol 2013;40:527-8.

30 Nakasa T, Miyaki S, Okubo A, et al. Expression of microRNA-146 in rheumatoid arthritis synovial tissue. Arthritis Rheum 2008;58:1284-92.

31 Blüml S, Bonelli M, Niederreiter B, et al. Essential role of microRNA-155 in the pathogenesis of autoimmune arthritis in mice. Arthritis Rheum 2011;63:1281-8.

32 Nakasa $T$, Shibuya $H$, Nagata $Y$, et al. The inhibitory effect of microRNA-146a expression on bone destruction in collagen-induced arthritis. Arthritis Rheum 2011;63:1582-90.

33 Li YT, Chen SY, Wang CR, et al. Amelioration of collagen-induced arthritis in mice by lentivirus-mediated silencing of microRNA-223. Arthritis Rheum 2012:64:3240-5

34 Vickers KC, Palmisano BT, Shoucri BM, et al. MicroRNAs are transported in plasma and delivered to recipient cells by high-density lipoproteins. Nat Cell Biol 2011;13:423-33.

35 Arroyo JD, Chevillet JR, Kroh EM, et al. Argonaute2 complexes carry a population of circulating microRNAs independent of vesicles in human plasma. Proc Natl Acad Sci USA 2011;108:5003-8.

36 Gilad S, Meiri E, Yogev $Y$, et al. Serum microRNAs are promising novel biomarkers. PLoS One 2008;3:e3148.

37 Wang JF, Yu ML, Yu G, et al. Serum miR-146a and miR-223 as potential new biomarkers for sepsis. Biochem Biophys Res Commun 2010;394:184-8.

38 Pigati $L$, Yaddanapudi SC, lyengar $R$, et al. Selective release of microRNA species from normal and malignant mammary epithelial cells. PLoS One 2010;5:e13515.

39 Iguchi $\mathrm{H}$, Kosaka N, Ochiya T. Secretory microRNAs as a versatile communication tool. Commun Integr Biol 2010:3:478-81.

40 Sebastiani GD, Fulci V, Niccolini S, et al. Over-expression of miR-223 in T-lymphocytes of early rheumatoid arthritis patients. Clin Exp Rheumatol 2011;29:1058-9.

41 Landgraf $\mathrm{P}$, Rusu M, Sheridan R, et al. A mammalian microRNA expression atlas based on small RNA library sequencing. Cell 2007;129:1401-14.

42 McInnes IB, Schett G. Cytokines in the pathogenesis of rheumatoid arthritis. Nat Rev Immunol 2007;7:429-42.

43 Ospelt C, Gay S. The role of resident synovial cells in destructive arthritis. Best Pract Res Clin Rheumatol 2008;22:239-52.

44 Raza K, Falciani F, Curnow SJ, et al. Early rheumatoid arthritis is characterized by a distinct and transient synovial fluid cytokine profile of $\mathrm{T}$ cell and stromal cell origin. Arthritis Res Ther 2005;7:R784-95. 